\title{
MULTI-DIMENSIONAL WEYL MODULES AND SYMMETRIC FUNCTIONS
}

\author{
B. FEIGIN, S. LOKTEV
}

\begin{abstract}
The Weyl modules in the sense of V. Chari and A. Pressley ([CP $)$ over the current Lie algebra on an affine variety are studied. We show that local Weyl modules are finite-dimensional and generalize the tensor product decomposition theorem from $[\mathrm{CP}$.

More explicit results are stated for currents on a non-singular affine variety of dimension $d$ with coefficients in the Lie algebra $s l_{r}$. The Weyl modules with highest weights proportional to the vector representation one are related to the multi-dimensional analogs of harmonic functions. The dimensions of such local Weyl modules are calculated in the following cases.

For $d=1$ we show that the dimensions are equal to powers of $r$. For $d=2$ we show that the dimensions are given by products of the higher Catalan numbers (the usual Catalan numbers for $r=2$ ). We finally formulate a conjecture for an arbitrary $d$ and $r=2$.
\end{abstract}

\section{INTRODUCTION}

Let $M$ be a complex affine variety, let $\mathfrak{g}$ be a semisimple Lie algebra. By $\mathfrak{g}^{M}$ denote the Lie algebra of currents on $M$. For any point $\mathcal{Z} \in M$ we have the evaluation homomorphism $\mathfrak{g}^{M} \rightarrow \mathfrak{g}$ taking value at $\mathcal{Z}$. So for any representation $\pi$ of $\mathfrak{g}$ we can define the evaluation representation $\pi(\mathcal{Z})$ of $\mathfrak{g}^{M}$.

Now let $\pi_{1}, \ldots, \pi_{n}$ be irreducible representations of $\mathfrak{g}$ and let $\mathcal{Z}_{1}, \ldots, \mathcal{Z}_{n}$ be points on $M$. Suppose that the points $\mathcal{Z}_{i}$ are pairwise distinct. Then $\pi_{1}\left(\mathcal{Z}_{1}\right) \otimes \cdots \otimes \pi_{n}\left(\mathcal{Z}_{n}\right)$ is an irreducible representation of $\mathfrak{g}^{M}$. Fix the highest weight vectors $v_{i} \in \pi_{i}$. Then the vector $v=v_{1} \otimes \cdots \otimes v_{n}$ is cyclic, therefore

$$
\pi_{1}\left(\mathcal{Z}_{1}\right) \otimes \cdots \otimes \pi_{n}\left(\mathcal{Z}_{n}\right) \cong U\left(\mathfrak{g}^{M}\right) / I\left(\pi_{1}, \ldots, \pi_{n} ; \mathcal{Z}_{1}, \ldots, \mathcal{Z}_{n}\right),
$$

where $I\left(\pi_{1}, \ldots, \pi_{n} ; \mathcal{Z}_{1}, \ldots, \mathcal{Z}_{n}\right)$ is the left ideal annihilating $v$. So we have a family $\mathcal{N}$ of ideals labeled by $M \times \cdots \times M$ without diagonals.

Let $\widetilde{\mathcal{N}}$ be the closure of $\mathcal{N}$ in the space of all left ideals in $U\left(\mathfrak{g}^{M}\right)$. Then $\widetilde{\mathcal{N}}$ is an algebraic variety (in most cases singular). Quotients of $U\left(\mathfrak{g}^{M}\right)$ by the corresponding ideals form a bundle $\xi$ on $\widetilde{\mathcal{N}}$ of $\operatorname{dimension} \operatorname{dim}\left(\pi_{1}\right) \cdots \operatorname{dim}\left(\pi_{n}\right)$.

The case $M=\mathbb{C}, \mathfrak{g}=s l_{2}$ is completely studied in $[\mathrm{FF}$. Here the limits of ideals are unique and the fiber over $(0, \ldots, 0)$ is isomorphic to the fusion product introduced in $[\mathrm{FL}$. Now suppose that all the representations are 2-dimensional. Then $\widetilde{\mathcal{N}}$ is just the $n$-th symmetric power of $\mathbb{C}$ and the fusion product is universal in the following sense. It is the maximal finite-dimensional module generated by the vector $v$ such that

$$
(e \otimes P) v=0, \quad(h \otimes P) v=n P(0) \cdot v,
$$

where $P$ is a polynomial, and $e, h, f$ constitute the standard basis in $s l_{2}$. Following $[\mathrm{CP}$ we call such representations Weyl modules.

We expect that for $M=\mathbb{C}^{d}, \mathfrak{g}=s l_{2}$ and 2-dimensional representations the variety $\widetilde{\mathcal{N}}$ is a general locus in the Hilbert scheme of $n$-points on $\mathbb{C}^{d}$. So there is the canonical projection $\theta: \widetilde{\mathcal{N}} \rightarrow S^{n}\left(\mathbb{C}^{d}\right)$. By $\widetilde{\mathcal{N}}_{0}$ denote the special fiber $\theta^{-1}(0, \ldots, 0)$. Then we expect that the space of sections of the $2^{d}$-dimensional bundle $\xi$ on $\widetilde{\mathcal{N}}_{0}$ has the same universality property.

Note that both facts can be derived for $d=2$ from the recent results of M. Haiman ( $\underline{\underline{H}}]$ ). It gives a very powerful instrument to study fusion and Weyl modules over two-dimensional currents.

In this paper we concentrate on the Weyl module side of this picture. In Section 1 we extend the notion of Weyl modules. Namely we define the global Weyl module corresponding to a commutative algebra with unit, a semisimple Lie algebra and a weight, as well as the local Weyl module corresponding to the data above and a set of points on Spec $A$. We show that local Weyl modules are finite-dimensional and generalize the tensor product theorem $([\mathrm{CP}]$ ) for them. In Section 2 we generalize the symmetric power construction proposed in [CP]. In Section 3 we apply the results of $[\mathrm{H}]$ to investigation of Weyl modules over two-dimensional currents and state a conjecture for the general case. 
Acknowledgments. This consideration is inspired by discussions with Vyjayanthi Chari at UC Riverside and MSRI. SL thanks V. Chari for the hospitality at UC Riverside. We are also grateful to V. V. Dotsenko, A. N. Kirillov and I. N. Nikokoshev for useful and stimulating discussions.

BF is partially supported by the grants RFBR-02-01-01015, RFBR-01-01-00906 and INTAS-00-00055.

SL is partially supported by the grants RFBR-02-01-01015, RFBR-01-01-00546 and CRDF RM1-2545-MO-03.

\section{Some Generalities}

1.1. Notation and integrability. Let $\mathfrak{g}$ be a semisimple Lie algebra of rank $r$. Choose a Cartan subalgebra $\mathfrak{h}$ and Borel subalgebras $\mathfrak{b}=\mathfrak{b}_{+}$and $\mathfrak{b}_{-}$. Denote their nilpotent radicals by $\mathfrak{n}_{+}$and $\mathfrak{n}_{-}$respectively.

By $Q \subset \mathfrak{h}^{*}$ denote the root lattice, by $P \subset \mathfrak{h}^{*}$ denote the weight lattice. Let $\alpha_{1}, \ldots, \alpha_{r} \in Q$ be the simple roots, let $\omega_{1}, \ldots, \omega_{r} \in P$ be the simple weights. Denote the correspondent Chevalley generators by $e_{1}, \ldots, e_{r} \in \mathfrak{n}_{+}$, $h_{1}, \ldots, h_{r} \in \mathfrak{h}$ and $f_{1}, \ldots, f_{r} \in \mathfrak{n}_{-}$. Let $Q_{+} \subset Q$ be the cone of positive roots, that is the set of linear combination of simple roots $\alpha_{i}$ with non-negative integer coefficients.

Definition 1. We say that a $\mathfrak{g}$-module $V$ is integrable if there exists a finite subset $D \subset P$ such that

$$
V=\bigoplus_{\mu \in D} V^{\mu}
$$

where any $h \in \mathfrak{h}$ acts on $V^{\mu}$ by the scalar $\mu(h)$.

Note that for our purposes we do not require $V^{\mu}$ to be finite-dimensional. Similar to the case of finite-dimensional weight spaces (see $[\underline{K}]$ ) it actually implies that $V$ is a possibly infinite sum of finite-dimensional representations.

Proposition 1. There is the action of the Weyl group on the weights of integrable representations.

Proof. It is enough (see $[\mathrm{K}]$ ) to introduce the action of the operators

$$
s_{i}=\exp \left(f_{i}\right) \exp \left(-e_{i}\right) \exp \left(f_{i}\right)
$$

corresponding to simple reflections. These operators are well-defined on an integrable representation because the action of $e_{i}$ and $f_{i}$ is nilpotent. So similar to the case of finite-dimensional representations it defines the action of the Weyl group on the weights.

Also we consider the following class of representation.

Definition 2. Given a weight $\lambda \in \mathfrak{h}^{*}$ we say that a $\mathfrak{g}$-module $V$ is $\lambda$-bounded if

$$
V=\bigoplus_{\mu \in \lambda-Q_{+}} V^{\mu}
$$

where any $h \in \mathfrak{h}$ acts on $V^{\mu}$ by the scalar $\mu(h)$.

This definition is related to the definition of the category $\mathcal{O}$ (see $[\mathrm{K}]$ ). Namely any representation from the category $\mathcal{O}$ is by definition a direct sum of $\lambda^{i}$-bounded modules for some $\lambda^{1}, \ldots, \lambda^{N} \in \mathfrak{h}^{*}$ with finite-dimensional weight spaces.

Proposition 2. Any $\lambda$-bounded $\mathfrak{g}$-module has the maximal integrable quotient.

Proof. Suppose that a module $V=\bigoplus V^{\mu}$ is $\lambda$-bounded. By $w_{0} \in W$ denote the highest length element, so $w_{0}\left(Q_{+}\right)=-Q_{+}$. Let $V^{\prime}$ be the submodule of $V$ generated by $V^{\mu}$ such that $\mu \notin w_{0}(\lambda)+Q_{+}$. Introduce the module $V^{i n t}=V / V^{\prime}$. As the intersection of $\lambda-Q_{+}$and $w_{0}(\lambda)+Q_{+}$is finite, the module $V^{\text {int }}$ is integrable.

Now let $U$ be an integrable quotient of $V$. Let us show that $U$ is indeed a quotient of $V^{\text {int }}$.

As $U$ is a quotient of $V$ we have $U^{\mu}=0$ if $\mu \notin \lambda-Q_{+}$. Then Proposition 1 implies that $U^{\mu}=0$ if $\mu \notin$ $w_{0}\left(\lambda-Q_{+}\right)=w_{0}(\lambda)+Q_{+}$. Therefore the image of $V^{\prime}$ in $U$ is zero, so $U$ is a quotient of $V^{\text {int }}$.

Let us say that a module with a certain property is maximal if any other module with this property is a quotient of the given one. 
1.2. Weyl modules. Let $A$ be a commutative algebra with unit. Generalizing $[\mathrm{CP}]$ we define a family of representations of the Lie algebra $\mathfrak{g} \otimes A$.

Definition 3. For $\lambda \in \mathfrak{h}^{*}$ define the global Weyl module $W_{A}(\lambda)$ as the maximal $\mathfrak{g} \otimes 1$-integrable module generated by the vector $v_{\lambda}$ such that

$$
\left(\mathfrak{n}_{+} \otimes P\right) v_{\lambda}=0, \quad(h \otimes 1) v_{\lambda}=\lambda(h) \cdot v_{\lambda} \quad \text { for } h \in \mathfrak{h}, P \in A .
$$

In particular if $A=\mathbb{C}\left[x, x^{-1}\right]$ then $W_{A}(\lambda) \cong W(\lambda)$ in the terms of [CP.

Proposition 3. The global Weyl module $W_{A}(\lambda)$ exists.

Proof. Let $\mathfrak{b}_{A} \subset \mathfrak{g} \otimes A$ be the subalgebra spanned by $\mathfrak{n}_{+} \otimes A$ and $\mathfrak{h} \otimes 1$. Then the induced module

$$
M_{A}(\lambda)=\operatorname{Ind}_{\mathfrak{b}_{A}}^{\mathfrak{g} \otimes A} v_{\lambda}
$$

is the maximal module generated by $v_{\lambda}$.

As $M_{A}(\lambda)$ is generated by $v_{\lambda}$ under the action of $U\left(\mathfrak{b}_{-} \otimes A\right)$, the module $M_{A}(\lambda)$ is $\lambda$-bounded. So we can define $W_{A}(\lambda)$ as the maximal integrable quotient of $M_{A}(\lambda)$.

Concerning the universality property, any integrable module generated by $v_{\lambda}$ is an integrable quotient of $M_{A}(\lambda)$ and therefore a quotient of the constructed module $W_{A}(\lambda)$.

Lemma 1. We have $W_{A}(\lambda) \neq 0$ if and only if $\lambda$ is dominant.

Proof. Note that $v_{\lambda}$ can generate an integrable module only for a dominant $\lambda$.

On the other hand, suppose $\lambda$ is dominant. Let $\pi_{\lambda}$ be the irreducible representation of $\mathfrak{g}$ with highest weight $\lambda$, let $v$ be its highest weight vector. Then $\pi_{\lambda} \otimes A$ is an integrable module over $\mathfrak{g} \otimes A$ and $v \otimes 1$ satisfies the conditions (1). So $W_{A}(\lambda)$ has a non-trivial quotient $\pi_{\lambda} \otimes A$, therefore $W_{A}(\lambda) \neq 0$.

Now regard $A$ as the algebra of functions on an affine variety $M$ (possibly singular). Actually points on $M$ are just ideals in $A$ of codimension one.

Now suppose that $\lambda$ is dominant. Then the coordinates $\lambda_{i}=\lambda\left(h_{i}\right)$ are non-negative integers. A set of points $\mathcal{Z}_{j}^{(i)} \in M$ (not necessarily distinct) indexed as follows:

$$
\{\mathcal{Z}\}_{\lambda}=\left(\left\{\mathcal{Z}_{j}^{(1)}\right\}_{j=1 \ldots \lambda_{1}}, \ldots,\left\{\mathcal{Z}_{j}^{(r)}\right\}_{j=1 \ldots \lambda_{r}}\right)
$$

we call a $\lambda$-multiset.

Definition 4. Let $\lambda=\left(\lambda_{1}, \ldots, \lambda_{r}\right) \in \mathfrak{h}^{*}$ be a dominant weight, let $\{\mathcal{Z}\}_{\lambda}$ be a $\lambda$-multiset. Define the local Weyl module $W_{M}\left(\{\mathcal{Z}\}_{\lambda}\right)$ as the maximal $\mathfrak{g} \otimes 1$-integrable module generated by the vector $v_{\{\mathcal{Z}\}_{\lambda}}$ such that

$$
\left(\mathfrak{n}_{+} \otimes P\right) v_{\{\mathcal{Z}\}_{\lambda}}=0, \quad\left(h_{i} \otimes P\right) v_{\{\mathcal{Z}\}_{\lambda}}=\sum_{j=1}^{\lambda_{i}} P\left(\mathcal{Z}_{j}^{(i)}\right) \cdot v_{\{\mathcal{Z}\}_{\lambda}} \quad \text { for } i=1 \ldots r, P \in A .
$$

In particular if $M=\mathbb{C}^{*}, A=\mathbb{C}\left[x, x^{-1}\right]$ then $W_{M}\left(\{\mathcal{Z}\}_{\lambda}\right) \cong W(\pi)$ in the terms of [CP for $\pi_{i}(u)=\prod_{j}\left(1-u / \mathcal{Z}_{j}^{(i)}\right)$. In general case this definition is motivated by Theorem 5 from Section 2.

Proposition 4. The local Weyl module $W_{M}\left(\{\mathcal{Z}\}_{\lambda}\right)$ exists and can be obtained as a quotient of the global Weyl module $W_{A}(\lambda)$.

Proof. Similarly to Proposition 3 one can define $W_{M}\left(\{\mathcal{Z}\}_{\lambda}\right)$ as the maximal integrable quotient of the induced module

$$
M_{M}\left(\{\mathcal{Z}\}_{\lambda}\right)=\operatorname{Ind}_{\mathfrak{b} \otimes A}^{\mathfrak{g} \otimes A} v_{\{\mathcal{Z}\}_{\lambda}} .
$$

As $\mathfrak{b}_{A}$ acts on $v_{\{\mathcal{Z}\}_{\lambda}}$ in the same way as on $v_{\lambda}$, the universality property implies that $W_{M}\left(\{\mathcal{Z}\}_{\lambda}\right)$ is a quotient of $W_{A}(\lambda)$.

Proposition 5. We have $W_{M}\left(\{\mathcal{Z}\}_{\lambda}\right) \neq 0$.

Proof. With any $\mathcal{Z} \in\{\mathcal{Z}\}_{\lambda}$ we associate the weight $\lambda_{\mathcal{Z}}$ such that $h_{i}\left(\lambda_{\mathcal{Z}}\right)$ is the multiplicity of $\mathcal{Z}$ in $\left\{\mathcal{Z}_{j}^{(i)}\right\}_{j=1 \ldots \lambda_{i}}$.

For $\mathcal{Z} \in M$ and a representation $\pi$ of $\mathfrak{g}$ let $\pi(\mathcal{Z})$ be the evaluation representation of $\mathfrak{g} \otimes A$ at $\mathcal{Z}$. Namely $\pi(\mathcal{Z})=\pi \otimes(A / I(\mathcal{Z}))$, where $I(\mathcal{Z})$ is the maximal ideal corresponding to the point $\mathcal{Z}$.

By $\pi_{\mu}$ denote the irreducible representation with highest weight $\mu$. Then the representation

$$
\bigotimes_{\mathcal{Z} \in\{\mathcal{Z}\}_{\lambda}} \pi_{\lambda_{\mathcal{Z}}}(\mathcal{Z})
$$


is integrable and the product of highest weight vectors satisfies (2). So $W_{M}\left(\{\mathcal{Z}\}_{\lambda}\right)$ has a non-trivial quotient, therefore $W_{M}\left(\{\mathcal{Z}\}_{\lambda}\right) \neq 0$.

At last note that the action of the Weyl group restricts weights of the Weyl modules as follows.

Lemma 2. We have $W_{A}(\lambda)^{\mu} \neq 0$ only for $\mu \in \bigcap_{w \in W} w\left(\lambda-Q_{+}\right)$, and the same statement for $W_{M}\left(\{\mathcal{Z}\}_{\lambda}\right)$.

Proof. We just combine the observation that $W_{A}(\lambda)$ and $W_{M}\left(\{\mathcal{Z}\}_{\lambda}\right)$ are $\lambda$-bounded and the action of the Weyl group on their weights.

\subsection{The Local Weyl modules are finite-dimensional.}

Lemma 3. We have $W_{A}(\lambda)^{\lambda-N \alpha_{i}} \neq 0$ only for $0 \leq N \leq \lambda_{i}$, and the same statement for $W_{M}\left(\{\mathcal{Z}\}_{\lambda}\right)$.

Proof. It follows from Lemma 2] For $N<0$ we have $\lambda-N \alpha_{i} \notin \lambda-Q_{+}$, for $N>\lambda_{i}$ we have $\lambda-N \alpha_{i} \notin s_{i}\left(\lambda-Q_{+}\right)$, where $s_{i}$ is the simple reflection.

Let $I\left(\{\mathcal{Z}\}_{\lambda}\right)$ be the ideal in $A$ of functions with zeroes at all $\mathcal{Z}_{j}^{(i)}$. In order to get multiple zeroes we consider the powers $I^{s}\left(\{\mathcal{Z}\}_{\lambda}\right)$.

Proposition 6. Elements of $f_{i} \otimes I^{\lambda_{i}}\left(\{\mathcal{Z}\}_{\lambda}\right)$ act on $v_{\{\mathcal{Z}\}_{\lambda}}$ by zero.

Proof. The ideal $I^{\lambda_{i}}\left(\{\mathcal{Z}\}_{\lambda}\right)$ is spanned by decomposable elements, that is by products of $\lambda_{i}$ elements of $I\left(\{\mathcal{Z}\}_{\lambda}\right)$. So it is enough to prove that if $P=P_{1} \cdots P_{\lambda_{i}}$ for $P_{i} \in I\left(\{\mathcal{Z}\}_{\lambda}\right)$ then $\left(f_{i} \otimes P\right) v_{\{\mathcal{Z}\}_{\lambda}}=0$.

From Lemma 3 it follows that $\left(f_{i} \otimes 1\right)^{\lambda_{i}+1} v_{\{\mathcal{Z}\}_{\lambda}}=0$. Then using Proposition [16] (see Appendix) we have

$$
0=\left(e_{i} \otimes P_{1}\right) \cdots\left(e_{i} \otimes P_{\lambda_{i}}\right) \cdot\left(f_{i} \otimes 1\right)^{\lambda_{i}+1} v_{\{\mathcal{Z}\}_{\lambda}}=C \cdot\left(f_{i} \otimes P\right) v_{\{\mathcal{Z}\}_{\lambda}},
$$

where the coefficient $C \neq 0$.

Proposition 7. For a large enough integer $N$ the ideal $\mathfrak{g} \otimes I^{N}\left(\{\mathcal{Z}\}_{\lambda}\right)$ acts on $W_{M}\left(\{\mathcal{Z}\}_{\lambda}\right)$ by zero.

Proof. Note that the Lie subalgebra $\mathfrak{n}_{-}$is generated by $f_{i}, i=1 \ldots r$. So $\mathfrak{n}_{-}$is spanned by a finite number of elements $x_{\left(i_{1}, \ldots, i_{s}\right)}=\operatorname{ad}\left(f_{i_{1}}\right) \cdots \operatorname{ad}\left(f_{i_{s-1}}\right) f_{i_{s}}$. Let us take

$$
N \geq \max _{\substack{\left(i_{1}, \ldots, i_{s}\right) \\ x_{\left(i_{1}, \ldots, i_{s}\right)} \neq 0}}\left(\lambda_{i_{1}}+\cdots+\lambda_{i_{s}}\right) .
$$

Then for a decomposable $P \in I^{N}\left(\{\mathcal{Z}\}_{\lambda}\right)$ and $\left(i_{1}, \ldots, i_{s}\right)$ such that $x_{\left(i_{1}, \ldots, i_{s}\right)} \neq 0$ we have $P=P_{1} \cdots P_{s}$, where $P_{j} \in I^{\lambda_{i_{j}}}\left(\{\mathcal{Z}\}_{\lambda}\right)$. From Proposition [6] we have $\left(f_{i_{j}} \otimes P_{j}\right) v\{\mathcal{Z}\}_{\lambda}=0$, so

$$
\left(x_{\left(i_{1}, \ldots, i_{s}\right)} \otimes P\right) v_{\{\mathcal{Z}\}_{\lambda}}=\left(\operatorname{ad}\left(f_{i_{1}} \otimes P_{1}\right) \cdots \operatorname{ad}\left(f_{i_{s-1}} \otimes P_{s-1}\right)\left(f_{i_{s}} \otimes P_{s}\right)\right) v_{\{\mathcal{Z}\}_{\lambda}}=0 .
$$

It means that $\mathfrak{n}_{-} \otimes I^{N}\left(\{\mathcal{Z}\}_{\lambda}\right)$ acts on $v\{\mathcal{Z}\}_{\lambda}$ by zero.

As $\mathfrak{b}_{+} \otimes I\left(\{\mathcal{Z}\}_{\lambda}\right)$ acts on $v_{\{\mathcal{Z}\}_{\lambda}}$ by zero and $\mathfrak{g}=\mathfrak{b}_{+} \oplus \mathfrak{n}_{-}$, we have $\left(\mathfrak{g} \otimes I^{N}\left(\{\mathcal{Z}\}_{\lambda}\right)\right) v_{\{\mathcal{Z}\}_{\lambda}}=0$. Finally note that $\mathfrak{g} \otimes I^{N}\left(\{\mathcal{Z}\}_{\lambda}\right)$ is an ideal in the Lie algebra $\mathfrak{g} \otimes A$, so we have the statement of the proposition.

Theorem 1. The local Weyl module $W_{M}\left(\{\mathcal{Z}\}_{\lambda}\right)$ is finite-dimensional.

Proof. Recall that $W_{M}\left(\{\mathcal{Z}\}_{\lambda}\right)$ is generated by $v_{\{\mathcal{Z}\}_{\lambda}}$ under the action of $\mathfrak{n}_{-} \otimes A$, in other words we have $W_{M}\left(\{\mathcal{Z}\}_{\lambda}\right)=$ $U\left(\mathfrak{n}_{-} \otimes A\right) v_{\{\mathcal{Z}\}_{\lambda}}$. From Proposition 7 we have that $W_{M}\left(\{\mathcal{Z}\}_{\lambda}\right)=U\left(\mathfrak{n}_{-} \otimes A / I^{N}\left(\{\mathcal{Z}\}_{\lambda}\right)\right) v_{\{\mathcal{Z}\}_{\lambda}}$ for a certain $N$.

Now let us deduce from integrability that there exists an integer $n$ such that $x_{1} \cdots x_{s} v_{\{\mathcal{Z}\}_{\lambda}}=0$ in $W_{M}\left(\{\mathcal{Z}\}_{\lambda}\right)$ for $s>n$ and any $x_{i} \in \mathfrak{n}_{-} \otimes A$.

Let $\rho^{\vee}$ be the element of $\mathfrak{h}$ such that $\rho^{\vee}\left(\alpha_{i}\right)=1$ for $i=1 \ldots r$. As $W_{M}\left(\{\mathcal{Z}\}_{\lambda}\right)$ is integrable, the set of values of $\rho^{\vee}$ on weights of $W_{M}\left(\{\mathcal{Z}\}_{\lambda}\right)$ is finite. Suppose that $x_{1} \cdots x_{s} v_{\{\mathcal{Z}\}_{\lambda}} \neq 0$, let $\mu$ be its weight. Then $\rho^{\vee}(\mu)<\rho^{\vee}(\lambda)-s$. So $s$ is not greater than the maximal difference between $\rho^{\vee}(\lambda)$ and $\rho^{\vee}(\mu)$, where $\mu$ is a weight of $W_{M}\left(\{\mathcal{Z}\}_{\lambda}\right)$.

Now we have

$$
W_{M}\left(\{\mathcal{Z}\}_{\lambda}\right)=U^{\leq n}\left(\mathfrak{n}_{-} \otimes A / I^{N}\left(\{\mathcal{Z}\}_{\lambda}\right)\right) v_{\{\mathcal{Z}\}_{\lambda}} .
$$

As the Lie algebra $\mathfrak{n}_{-} \otimes A / I^{N}\left(\{\mathcal{Z}\}_{\lambda}\right)$ is finite-dimensional, this space is also finite-dimensional. 
1.4. Tensor product decomposition. For two multisets $\{\mathcal{Z}\}_{\lambda}$ and $\left\{\mathcal{Z}^{\prime}\right\}_{\lambda^{\prime}}$ define the union multiset

$$
\left\{\mathcal{Z} \cup \mathcal{Z}^{\prime}\right\}_{\lambda+\lambda^{\prime}}=\left(\left\{\mathcal{Z}_{j}^{(1)}\right\} \cup\left\{\left(\mathcal{Z}^{\prime}\right)_{j}^{(1)}\right\}, \ldots,\left\{\mathcal{Z}_{j}^{(r)}\right\} \cup\left\{\left(\mathcal{Z}^{\prime}\right)_{j}^{(r)}\right\}\right) .
$$

Lemma 4. We have an isomorphism of 1-dimensional representations of $\mathfrak{b} \otimes A$

$$
v_{\left\{\mathcal{Z} \cup \mathcal{Z}^{\prime}\right\}_{\lambda+\lambda^{\prime}}} \cong v_{\{\mathcal{Z}\}_{\lambda}} \bigotimes v_{\left\{\mathcal{Z}^{\prime}\right\}_{\lambda^{\prime}}} .
$$

Proof. It follows from the definition (2).

Proposition 8. Let $A_{N\{\mathcal{Z}\}_{\lambda}}=A / I_{\{\mathcal{Z}\}_{\lambda}}^{N}$. Suppose that $\mathcal{Z}_{j}^{(i)} \neq\left(\mathcal{Z}^{\prime}\right)_{j^{\prime}}^{\left(i^{\prime}\right)}$ for all $i, j, i^{\prime}, j^{\prime}$. Then we have an isomorphism of algebras

$$
A_{N\left\{\mathcal{Z} \cup \mathcal{Z}^{\prime}\right\}_{\lambda+\lambda^{\prime}}} \cong A_{N\{\mathcal{Z}\}_{\lambda}} \bigoplus A_{N\left\{\mathcal{Z}^{\prime}\right\}_{\lambda^{\prime}}} .
$$

Proof. From the Hilbert Nullstellensatz we have that $I_{\left\{\mathcal{Z} \cup \mathcal{Z}^{\prime}\right\}_{\lambda+\lambda^{\prime}}}^{N}=I_{\{\mathcal{Z}\}_{\lambda}}^{N} \cap I_{\left\{\mathcal{Z}^{\prime}\right\}_{\lambda^{\prime}}}^{N}$ and that $I_{\{\mathcal{Z}\}_{\lambda}}^{N}+I_{\left\{\mathcal{Z}^{\prime}\right\}_{\lambda^{\prime}}}^{N}=A$. Then the direct sum of the natural projections

$$
A \rightarrow A_{N\{\mathcal{Z}\}_{\lambda}} \bigoplus A_{N\left\{\mathcal{Z}^{\prime}\right\}_{\lambda^{\prime}}}
$$

is surjective and its kernel is equal to $I_{\left\{\mathcal{Z} \cup \mathcal{Z}^{\prime}\right\}_{\lambda+\lambda^{\prime}}}^{N}$.

Theorem 2. Suppose that $\mathcal{Z}_{j}^{(i)} \neq\left(\mathcal{Z}^{\prime}\right)_{j^{\prime}}^{\left(i^{\prime}\right)}$ for all $i, j, i^{\prime}, j^{\prime}$. Then we have an isomorphism of $\mathfrak{g} \otimes A$-modules

$$
W_{M}\left(\left\{\mathcal{Z} \cup \mathcal{Z}^{\prime}\right\}_{\lambda+\lambda^{\prime}}\right) \cong W_{M}\left(\{\mathcal{Z}\}_{\lambda}\right) \bigotimes W_{M}\left(\left\{\mathcal{Z}^{\prime}\right\}_{\lambda^{\prime}}\right) \text {. }
$$

Proof. By Lemma 4 we have that the product $W_{M}\left(\{\mathcal{Z}\}_{\lambda}\right) \otimes W_{M}\left(\left\{\mathcal{Z}^{\prime}\right\}_{\lambda^{\prime}}\right)$ is a quotient of $W_{M}\left(\left\{\mathcal{Z} \cup \mathcal{Z}^{\prime}\right\}_{\lambda+\lambda^{\prime}}\right)$. Let us show the equality of their dimensions.

Right now we know that the dimension of $W_{M}\left(\left\{\mathcal{Z} \cup \mathcal{Z}^{\prime}\right\}_{\lambda+\lambda^{\prime}}\right)$ is not less than the dimension of the tensor product. Let us obtain the opposite inequality.

Recall the notation $A_{N\{\mathcal{Z}\}_{\lambda}}=A / I_{\{\mathcal{Z}\}_{\lambda}}^{N}$ and consider the corresponding induced module

$$
M_{M}^{N}\left(\{\mathcal{Z}\}_{\lambda}\right)=\operatorname{Ind}_{\mathfrak{b} \otimes A_{N\{\mathcal{Z}\}_{\lambda}}}^{\mathfrak{g} \otimes A_{N\{\mathcal{Z}}} v_{\{\mathcal{Z}\}_{\lambda}} .
$$

From Proposition $\left[\right.$ we know that $W_{M}\left(\{\mathcal{Z}\}_{\lambda}\right)$ is a quotient of $M_{M}^{N}\left(\{\mathcal{Z}\}_{\lambda}\right)$ for a sufficiently large $N$. Moreover as $M_{M}^{N}\left(\{\mathcal{Z}\}_{\lambda}\right)$ is a quotient of $M_{M}\left(\{\mathcal{Z}\}_{\lambda}\right)$, we have that $W_{M}\left(\{\mathcal{Z}\}_{\lambda}\right)$ is its maximal integrable quotient.

Lemma 4 with Proposition 8 implies that for any positive integer $N$ we have

$$
M_{M}^{N}\left(\left\{\mathcal{Z} \cup \mathcal{Z}^{\prime}\right\}_{\lambda+\lambda^{\prime}}\right) \cong M_{M}^{N}\left(\{\mathcal{Z}\}_{\lambda}\right) \otimes M_{M}^{N}\left(\left\{\mathcal{Z}^{\prime}\right\}_{\lambda^{\prime}}\right) .
$$

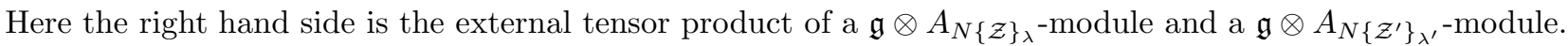

Now take a sufficiently large $N$ so three our $W_{M}$-s are quotients of the corresponding $M_{M}^{N}$-s. Then the module $W_{M}\left(\left\{\mathcal{Z} \cup \mathcal{Z}^{\prime}\right\}_{\lambda+\lambda^{\prime}}\right)$ is a quotient of the tensor product $M_{M}^{N}\left(\{\mathcal{Z}\}_{\lambda}\right) \otimes M_{M}^{N}\left(\left\{\mathcal{Z}^{\prime}\right\}_{\lambda^{\prime}}\right)$.

Concerning the weight subspaces, let us show that the image of $M_{M}^{N}\left(\{\mathcal{Z}\}_{\lambda}\right)^{\mu} \otimes M_{M}^{N}\left(\left\{\mathcal{Z}^{\prime}\right\}_{\lambda^{\prime}}\right)^{\mu^{\prime}}$ in the quotient $W_{M}\left(\left\{\mathcal{Z} \cup \mathcal{Z}^{\prime}\right\}_{\lambda+\lambda^{\prime}}\right)$ is zero except a finite number of $\mu$ and $\mu^{\prime}$.

Let $D$ be the set of weights of the module $W_{M}\left(\left\{\mathcal{Z} \cup \mathcal{Z}^{\prime}\right\}_{\lambda+\lambda^{\prime}}\right)$. As this module is integrable, $D$ is finite. Then the sets

$$
D_{1}=\left(\lambda-Q_{+}\right) \bigcap\left(-\lambda^{\prime}+D+Q_{+}\right), \quad D_{2}=\left(\lambda^{\prime}-Q_{+}\right) \bigcap\left(-\lambda+D+Q_{+}\right)
$$

are also finite. As the module $M_{M}^{N}\left(\{\mathcal{Z}\}_{\lambda}\right)$ is $\lambda$-bounded and $M_{M}^{N}\left(\left\{\mathcal{Z}^{\prime}\right\}_{\lambda^{\prime}}\right)$ is $\lambda^{\prime}$-bounded, the image of $M_{M}^{N}\left(\{\mathcal{Z}\}_{\lambda}\right)^{\mu} \otimes$ $M_{M}^{N}\left(\left\{\mathcal{Z}^{\prime}\right\}_{\lambda^{\prime}}\right)^{\mu^{\prime}}$ in $W_{M}\left(\left\{\mathcal{Z} \cup \mathcal{Z}^{\prime}\right\}_{\lambda+\lambda^{\prime}}\right)$ is zero unless $\lambda-\mu \in Q_{+}, \lambda^{\prime}-\mu^{\prime} \in Q_{+}$and, of course, $\mu+\mu^{\prime} \in D$. So it is not zero only if $\mu \in D_{1}$ and $\mu^{\prime} \in D_{2}$.

Let $M_{M}^{N}\left(\{\mathcal{Z}\}_{\lambda}\right)^{\prime}$ be the submodule of $M_{M}^{N}\left(\{\mathcal{Z}\}_{\lambda}\right)$ generated by weight subspaces $M_{M}^{N}\left(\{\mathcal{Z}\}_{\lambda}\right)^{\mu}$ with $\mu \notin D_{1}$. Consider the quotient $W_{M}^{N}\left(\{\mathcal{Z}\}_{\lambda}\right)=M_{M}^{N}\left(\{\mathcal{Z}\}_{\lambda}\right) / M_{M}^{N}\left(\{\mathcal{Z}\}_{\lambda}\right)^{\prime}$. Let $W_{M}^{N}\left(\left\{\mathcal{Z}^{\prime}\right\}_{\lambda^{\prime}}\right)$ be the similar quotient of $M_{M}^{N}\left(\left\{\mathcal{Z}^{\prime}\right\}_{\lambda^{\prime}}\right)$ with weights in $D_{2}$. Then we know that the module $W_{M}\left(\left\{\mathcal{Z} \cup \mathcal{Z}^{\prime}\right\}_{\lambda+\lambda^{\prime}}\right)$ is a quotient of $W_{M}^{N}\left(\{\mathcal{Z}\}_{\lambda}\right) \otimes W_{M}^{N}\left(\left\{\mathcal{Z}^{\prime}\right\}_{\lambda^{\prime}}\right)$.

Because the sets $D_{1}$ and $D_{2}$ are finite, the modules $W_{M}^{N}\left(\{\mathcal{Z}\}_{\lambda}\right)$ and $W_{M}^{N}\left(\left\{\mathcal{Z}^{\prime}\right\}_{\lambda^{\prime}}\right)$ are integrable. Then the universality property implies that the module $W_{M}\left(\left\{\mathcal{Z} \cup \mathcal{Z}^{\prime}\right\}_{\lambda+\lambda^{\prime}}\right)$ is indeed a quotient of $W_{M}\left(\{\mathcal{Z}\}_{\lambda}\right) \otimes W_{M}\left(\left\{\mathcal{Z}^{\prime}\right\}_{\lambda^{\prime}}\right)$. So

$$
\operatorname{dim}\left(W_{M}\left(\{\mathcal{Z}\}_{\lambda}\right) \bigotimes W_{M}\left(\left\{\mathcal{Z}^{\prime}\right\}_{\lambda^{\prime}}\right)\right) \geq \operatorname{dim} W_{M}\left(\left\{\mathcal{Z} \cup \mathcal{Z}^{\prime}\right\}_{\lambda+\lambda^{\prime}}\right)
$$

that completes the proof.

Corollary 1. Suppose that $M$ is non-singular of dimension $d$. Then any local Weyl module is isomorphic as a vector space to the tensor product of modules $W_{\mathbb{C}^{d}}\left(\{0\}_{\lambda^{i}}\right)$ for some dominant $\lambda^{1}, \ldots, \lambda^{s}$. 
Proof. By Theorem 2 it is enough to describe $W_{M}\left(\{\mathcal{Z}\}_{\lambda}\right)$ when $\{\mathcal{Z}\}_{\lambda}$ contains only one point $Z \in M$. In this case $I\left(\{\mathcal{Z}\}_{\lambda}\right)$ is the ideal $I_{Z}$ of functions with zero at $Z$. By Proposition 7 we know that starting from a certain $N$ $W_{M}\left(\{\mathcal{Z}\}_{\lambda}\right)$ depends only on $A / I^{N}\left(\{\mathcal{Z}\}_{\lambda}\right)$. And the algebra $A / I_{Z}^{N}$ is the same for any non-singular point on any affine variety of given dimension.

To proceed with a singular variety $M$ we need also to describe local Weyl modules at singular points.

\section{The SyMmetric POWER CONSTRUCTION}

Here we generalize the results of Section 6 in $\mathrm{CP}$. Our proofs are based on the following lemma.

Lemma 5. Let $X$ be a vector space with a basis $v_{i}, i \in I$. Suppose that the set $I$ is partially ordered such that any chain $i_{1}>i_{2}>i_{3}>\ldots$ is finite.

(i) Let $\left\{u_{i}\right\}, i \in I$, be a set of vectors such that each $u_{j}$ is the sum of $v_{j}$ and a linear combination of $v_{i}$ with $i<j$. Then the set $\left\{u_{i}\right\}$ is a basis of $X$.

(ii) Let $Y \subset X$ be a subspace. Suppose we have a subset $J \subset I$ such that for any $j \in J$ the vector $v_{j}$ plus a linear combination of $v_{i}$ with $i<j$ belongs to $Y$. Then the images of vectors $v_{i}$ for $i \notin J$ span $X / Y$.

Proof. To prove (i) it is enough to show that each $v_{j}$ is a linear combination of $u_{i}$ as well. The vector $v_{j}$ is equal to $u_{j}$ plus (maybe) a linear combination of some $v_{j_{a}^{\prime}}$ with $j_{a}^{\prime}<j$. Similarly each $v_{j_{a}^{\prime}}$ is equal to $u_{j_{a}^{\prime}}$ plus (maybe) a linear combination of some $v_{j_{a b}^{\prime \prime}}$ with $j_{a b}^{\prime \prime}<j_{a}^{\prime}$ and so on. As any decreasing chain is finite, this procedure is also finite, and the result is a linear combination of $u_{i}$.

To show (ii) let us take $u_{i}=v_{i}$ for $i \notin J$ and $u_{i}=v_{i}+\cdots \in Y$ for $i \in J$. Then by (i) the set $\left\{u_{i}\right\}$ is a basis, so any vector from $X$ is a linear combination of some $v_{i}$ for $i \notin J$ and a vector from $Y$.

2.1. The $s l_{2}$ case. For $\mathfrak{g}=s l_{2}$ let us write $e, h, f$ instead of $e_{1}, h_{1}, f_{1}$.

Let $V$ be the vector (2-dimensional) representation of $s l_{2}$. Then the vector space $L_{A}(V)=V \otimes A$ inherits the action of $s l_{2} \otimes A$. Following $\left[\mathrm{CP}\right.$ we consider the symmetric powers $S^{n}\left(L_{A}(V)\right)$.

Choose a basis $\{u, v\}$ in $V$ such that $h v=v, h u=-u$. Then we can decompose $S^{n}\left(L_{A}(V)\right)$ into eigenspaces of $h \otimes 1$.

Proposition 9. We have

$$
S^{n}\left(L_{A}(V)\right) \cong \bigoplus_{i=0}^{n} S^{n}\left(L_{A}(V)\right)^{n-2 i}, \quad \text { where } S^{n}\left(L_{A}(V)\right)^{n-2 i} \cong S^{i}(A) \bigotimes S^{n-i}(A) \quad \text { for } i=0 \ldots n
$$

and $h \otimes 1$ acts on $S^{n}\left(L_{A}(V)\right)^{s}$ by the scalar $s$.

Proof. We have

$$
S^{n}\left(L_{A}(V)\right) \cong S^{n}((u \otimes A) \bigoplus(v \otimes A)) \cong \bigoplus_{i=0}^{n} S^{i}(u \otimes A) \bigotimes S^{n-i}(v \otimes A) \cong \bigoplus_{i=0}^{n} S^{i}(A) \bigotimes S^{n-i}(A)
$$

Let

$$
v^{(n)}=(v \otimes 1) \bigotimes \cdots \bigotimes(v \otimes 1)
$$

This vector is cyclic in the module $S^{n}\left(L_{A}(V)\right)$. Now choose a basis $\left\{P_{i}\right\}$ in $A$ such that $P_{1}=1$. To avoid cumbersome notation let us write $g P_{i}$ instead of $g \otimes P_{i}$ and $g$ instead of $g \otimes 1$ for $g \in s l_{2}$.

Proposition 10. Monomials

$$
f P_{j_{1}} \cdots f P_{j_{i}} \cdot h P_{j_{i+1}} \cdots h P_{j_{n}} v^{(n)}, \quad j_{1} \geq \cdots \geq j_{i}, \quad j_{i+1} \geq \cdots \geq j_{n}, \quad i=0 \ldots n
$$

form a basis in $S^{n}\left(L_{A}(V)\right)$.

Proof. By definition the elements

$$
s_{j_{1}, \ldots, j_{n}}^{i}=\operatorname{Sym}\left(\left(u \otimes P_{j_{1}}\right) \bigotimes \cdots \bigotimes\left(u \otimes P_{j_{i}}\right) \bigotimes\left(v \otimes P_{j_{i+1}}\right) \bigotimes \cdots \bigotimes\left(v \otimes P_{j_{n}}\right)\right)
$$

for $j_{1} \geq \cdots \geq j_{i}, j_{i+1} \geq \cdots \geq j_{n}$ and $i=0 \ldots n$ form a basis of $S^{n}\left(L_{A}(V)\right)$.

Introduce the partial order on the vectors $\left(j_{1}, \ldots, j_{n}\right)$ that compares the number of $j_{k}$ equal to one for $k>i$ :

$$
\left(j_{1}, \ldots, j_{n}\right)>\left(j_{1}^{\prime}, \ldots, j_{n}^{\prime}\right) \quad \text { if } \quad\left|\left\{k>i \mid j_{k}=1\right\}\right|<\left|\left\{k>i \mid j_{k}^{\prime}=1\right\}\right| .
$$

Clearly it satisfies the condition of Lemma 5 
Then we have

$$
f P_{j_{1}} \cdots f P_{j_{i}} \cdot h P_{j_{i+1}} \cdots h P_{j_{n}} v^{(n)}=n ! \cdot s_{j_{1}, \ldots, j_{n}}^{i}+\text { lower terms, }
$$

where lower terms are given by a linear combination of $s_{j_{1}^{\prime}, \ldots, j_{n}^{\prime}}^{i}$ with $\left(j_{1}^{\prime}, \ldots, j_{n}^{\prime}\right)<\left(j_{1}, \ldots, j_{n}\right)$. Namely the main term appears when the factors in the left hand side are applied to pairwise distinct factors of $v^{(n)}$, and lower terms appear when some factors in the left hand side are applied to the same factor of $v^{(n)}$.

By Lemma [5 (i) we have the statement of the proposition.

Proposition 11. The global Weyl module $W_{A}(n \omega)$ is spanned by elements

$$
f P_{j_{1}} \cdots f P_{j_{i}} \cdot h P_{j_{i+1}} \cdots h P_{j_{n}} v_{n \omega}, \quad j_{1} \geq \cdots \geq j_{i}, \quad j_{i+1} \geq \cdots \geq j_{n}, \quad i=0 \ldots n .
$$

Proof. The PBW Theorem and Lemma 3 imply that $W_{A}(n \omega)$ is spanned by elements

$$
m_{j_{1}, \ldots, j_{s}}^{i}=f P_{j_{1}} \cdots f P_{j_{i}} \cdot h P_{j_{i+1}} \cdots h P_{j_{s}} v_{n \omega}, \quad j_{1} \geq \cdots \geq j_{i}, \quad j_{i+1} \geq \cdots \geq j_{s}, \quad i \leq n .
$$

We need to show that this is still true under the restriction $s \leq n$. Then by setting $j_{s+1}=\cdots=j_{n}=1$ (that is $P_{j}=1$ for $j>s$ ) we obtain the statement of the proposition.

Now fix the grading, that is fix the integer $i$. Introduce the partial order

$$
\left(j_{1}, \ldots, j_{s}\right)>\left(j_{1}^{\prime}, \ldots, j_{s^{\prime}}^{\prime}\right) \text { if } s>s^{\prime} \text { or }\left(s=s^{\prime} \text { and }\left|\left\{k \leq i \mid j_{k}=1\right\}\right|<\left|\left\{k \leq i \mid j_{k}^{\prime}=1\right\}\right|\right) .
$$

Clearly it satisfies the condition of Lemma 5

To show that the monomials (3) span $W_{A}(n \omega)$ let us just obtain the monomials (4) with $s>n$ as the highest terms of some relations in $W_{A}(n \omega)$.

As $e P \cdot v_{n \omega}=0$ for all $P \in A$, we have

$$
e P \cdot m_{j_{1}, \ldots, j_{s}}^{i}=\sum_{k=1}^{i} f P_{j_{1}} \ldots f P_{j_{k-1}} f P_{j_{k+1}} \ldots f P_{j_{i}} h\left(P P_{j_{k}}\right) h P_{j_{i+1}} \ldots h P_{s} v_{n \omega}+\text { lower length terms }
$$

where lower length terms are given by a linear combination of $m_{j_{1}^{\prime}, \ldots, j_{s^{\prime}}^{\prime}}^{i^{\prime}}$ with $s^{\prime}<s$. Therefore we have

$$
e P_{j_{i+1}} \cdots e P_{j_{s}} \cdot f^{s-i} \cdot f P_{j_{1}} \cdots f P_{j_{i}} v_{n \omega}=(s-i) ! m_{j_{1}, \ldots, j_{s}}^{i}+\text { lower terms, }
$$

where lower terms are given by a linear combination of $m_{j_{1}^{\prime}, \ldots, j_{s^{\prime}}^{\prime}}^{i^{\prime}}$ with $\left(j_{1}^{\prime}, \ldots, j_{s^{\prime}}^{\prime}\right)<\left(j_{1}, \ldots, j_{s}\right)$.

Now let $s>n$. Then from Lemma 3 we have $f^{s-i} \cdot f P_{j_{1}} \cdots f P_{j_{i}} v_{n \omega}=0$, therefore the left hand side of (5) is a relation in $W_{A}(n \omega)$ and $m_{j_{1}, \ldots, j_{s}}^{i}$ is the highest term of this relation.

Let $X$ be the formal span of monomials (4) and let $Y$ be the kernel of the natural map $X \rightarrow W_{A}(n \omega)$. Then Lemma 5 (ii) implies the statement of the proposition.

Theorem 3. We have the natural isomorphism of $s l_{2} \otimes A$-modules

$$
W_{A}(n \omega) \cong S^{n}\left(L_{A}(V)\right) \text {. }
$$

Proof. Note that $v^{(n)}$ satisfies (1), and that $S^{n}\left(L_{A}(V)\right)$ is generated by $v^{(n)}$. Then from the universality property we have the surjective map $W_{A}(n \omega) \rightarrow S^{n}\left(L_{A}(V)\right)$ sending $v_{n \omega}$ to $v^{(n)}$. Propositions 10] and 11 imply that this map is an isomorphism.

Note that the action of $A$ on itself gives us the action of $S^{n}(A)$ on $S^{n}\left(L_{A}(V)\right)$ commuting with the action of $s l_{2} \otimes A$. For a multiset $\{\mathcal{Z}\}_{n \omega}$ define the ideal

$$
J_{\{\mathcal{Z}\}_{n \omega}}=\left\{R \in S^{n}(A) \mid R\left(\mathcal{Z}_{1}^{(1)}, \ldots, \mathcal{Z}_{n}^{(1)}\right)=0\right\} .
$$

Theorem 4. For any nw-multiset $\{\mathcal{Z}\}_{n \omega}$ we have the natural isomorphism of $s_{2} \otimes$ A-modules

$$
W_{M}\left(\{\mathcal{Z}\}_{n \omega}\right) \cong S^{n}\left(L_{A}(V)\right) / J_{\{\mathcal{Z}\}_{n \omega}} \cdot S^{n}\left(L_{A}(V)\right) \text {. }
$$

Proof. From Theorem 3 and the universality property we have a surjective map $S^{n}\left(L_{A}(V)\right) \rightarrow W_{M}\left(\{\mathcal{Z}\}_{n \omega}\right)$ sending $v^{(n)}$ to $v_{\{\mathcal{Z}\}_{n \omega}}$.

First let us show that the image of $J_{\{\mathcal{Z}\}_{n \omega}} \cdot S^{n}\left(L_{A}(V)\right)$ under this map is zero. As the action of $S^{n}(A)$ commutes with the action of $\mathfrak{g} \otimes A$, it is enough to show that the image of $J_{\{\mathcal{Z}\}_{n \omega}} \cdot v^{(n)}$ is zero.

Note that the tensor algebra $T^{*}(A) \cong U(h \otimes A)$ acts on $v^{(n)}$. It defines the surjective map

$$
T^{*}(A) \rightarrow S^{n}\left(L_{A}(V)\right)^{\lambda} \cong S^{n}(A): \quad P_{1} \bigotimes \cdots \bigotimes P_{s} \rightarrow h P_{1} \cdots h P_{s} v^{(n)}
$$

As the action of $U(h \otimes A)$ commutes with the action of $S^{n}(A)$, this map is a homomorphism of algebras. Then we can describe it explicitly observing that the generators $P \in A=T^{1}(A)$ are mapped to $\operatorname{Sym}(P \otimes 1 \otimes \cdots \otimes 1)$. 
Now consider the augmentation $\varepsilon_{\{\mathcal{Z}\}_{n \omega}}$ of $S^{n}(A)$ defined by

$$
\varepsilon_{\{\mathcal{Z}\}_{n \omega}}(R)=R\left(\mathcal{Z}_{1}^{(1)}, \ldots, \mathcal{Z}_{n}^{(1)}\right) .
$$

We can pull it back to the augmentation $\epsilon_{\{\mathcal{Z}\}_{n \omega}}$ of $T^{*}(A)$. Then for the generators $P \in A$ we have

$$
\epsilon_{\{\mathcal{Z}\}_{n \omega}}(P)=\sum_{j=1}^{n} P\left(\mathcal{Z}_{j}^{(1)}\right) .
$$

As this is exactly the action of $h P \in h \otimes A$ on $v_{\{\mathcal{Z}\}_{n \omega}}$, the augmentation ideal $J_{\{\mathcal{Z}\}_{n \omega}}$ acts on the image of $v^{(n)}$ in $W_{M}\left(\{\mathcal{Z}\}_{n \omega}\right)$ by zero.

Now we have the surjective map $\phi: S^{n}\left(L_{A}(V)\right) / J_{\{\mathcal{Z}\}_{n \omega}} \cdot S^{n}\left(L_{A}(V)\right) \rightarrow W_{M}\left(\{\mathcal{Z}\}_{n \omega}\right)$. The module in the left hand side is generated by the image $v^{\prime}$ of $v^{(n)} \in S^{n}\left(L_{A}(V)\right)$. Note that $v^{\prime}$ satisfies the same conditions (2) as $v_{\{\mathcal{Z}\}_{n \omega}}$, namely $e \otimes A$ acts on $v^{(n)}$ and therefore on $v^{\prime}$ by zero, and $h \otimes A$ acts on $v^{\prime}$ by the augmentation (6). As $\phi\left(v^{\prime}\right)=v_{\{\mathcal{Z}\}_{n \omega}}$, the universality property of $W_{M}\left(\{\mathcal{Z}\}_{n \omega}\right)$ implies that $\phi$ is an isomorphism.

Proposition 12. Any quotient $W$ of $W_{A}(n \omega)$ such that $\operatorname{dim} W^{\mathfrak{n} \omega}=1$ is indeed a quotient of $W_{M}\left(\{\mathcal{Z}\}_{n \omega}\right)$ for a certain $n \omega$-multiset $\{\mathcal{Z}\}_{n \omega}$.

Proof. Let $v$ be the image of $v_{n \omega}$ in $W$. We know that $h \otimes A$ acts on $v$ by a certain functional on $A$, so the tensor algebra $T^{*}(A) \cong U(h \otimes A)$ acts on $v$ by a certain augmentation $\epsilon$.

Note that the defined above map $T^{*}(A) \rightarrow S^{n}(A)$ is surjective and that its kernel is contained in the augmentation ideal of $\epsilon$. Therefore $\epsilon$ is the pull back of a certain augmentation $\varepsilon$ of $S^{n}(A)$. It means that $S^{n}(A)$ acts on $v$ by $\varepsilon$. We have $\varepsilon \neq 0$ as the action of $1 \in S^{n}(A)$ on $v$ is not zero.

So the kernel of $\varepsilon$ is a maximal ideal in $S^{n}(A)$, i.e. a point on $S^{n}(M)$, that is a set of $n$ points on $M$ (not necessarily distinct). Denote them by $\mathcal{Z}_{j}^{(1)}, j=1 \ldots n$. Then the vector $v$ satisfies the same conditions (2) as $v_{\{\mathcal{Z}\}_{n \omega}}$. Universality property implies the proposition.

Theorem 5. Let $\mathfrak{g}$ be an arbitrary semisimple Lie algebra. Then any quotient $W$ of $W_{A}(\lambda)$ such that $\operatorname{dim} W^{\lambda}=1$ is indeed a quotient of $W_{M}\left(\{\mathcal{Z}\}_{\lambda}\right)$ for a certain $\lambda$-multiset $\{\mathcal{Z}\}_{\lambda}$.

Proof. Let $v$ be the image of $v_{\lambda}$ in $W$. For $i=1 \ldots r$ by $s l_{2}^{(i)}$ denote the subalgebra of $\mathfrak{g}$ generated by $e_{i}, h_{i}, f_{i}$.

Let $W^{(i)}$ be the subspace of $W$ generated by $v$ under the action of $s l_{2}^{(i)} \otimes A$. Then $W^{(i)}$ satisfies the conditions of Proposition [12 So $h_{i} \otimes A$ acts on $v$ according to (2) for a certain set $\left\{\mathcal{Z}_{j}^{(i)}\right\}_{j=1 \ldots \lambda_{i}}$. Combining these sets for $i=1 \ldots r$ into a $\lambda$-multiset, we obtain the statement of the theorem.

2.2. Beyond $s l_{2}$. Let us first consider the case $\mathfrak{g}=s l_{r+1}$. By $e_{i j}, f_{i j}, 1 \leq i<j \leq r+1$ denote the matrix units corresponding to positive and negative roots respectively.

Let $V_{r+1}$ be the vector representation. We can similarly define $L_{N}\left(V_{r+1}\right)=V_{r+1} \otimes A$ and consider the symmetric powers.

Proposition 13. We have

$$
\begin{gathered}
S^{n}\left(L_{A}\left(V_{r+1}\right)\right) \cong \bigoplus_{\substack{i_{0}+i_{1}+\cdots+i_{r}=n \\
i_{j} \geq 0}} S^{n}\left(L_{A}\left(V_{r+1}\right)\right)^{\left(i_{0}-i_{1}, i_{1}-i_{2}, \ldots, i_{r-1}-i_{r}\right)}, \text { where } \\
S^{n}\left(L_{A}\left(V_{r+1}\right)\right)^{\left(i_{0}-i_{1}, i_{1}-i_{2}, \ldots, i_{r-1}-i_{r}\right)} \cong S^{i_{0}}(A) \bigotimes \cdots \bigotimes S^{i_{r}}(A)
\end{gathered}
$$

and $h_{j} \otimes 1$ acts on $S^{n}\left(L_{A}\left(V_{r+1}\right)\right)^{\left(s_{1}, \ldots, s_{r}\right)}$ by the scalar $s_{j}$.

Proof. Similar to Proposition 9

Theorem 6. We have the natural isomorphism of $s l_{r+1} \otimes A$-modules

$$
W_{A}\left(n \omega_{1}\right) \cong S^{n}\left(L_{A}\left(V_{r+1}\right)\right) \text {. }
$$

Proof. Similar to Theorem 3 The basis is formed by monomials

$$
\left(f_{12} P_{j_{1}^{1}} \cdots f_{12} P_{j_{i_{1}}^{1}}\right) \cdots\left(f_{1, r+1} P_{j_{1}^{r}} \cdots f_{1, r+1} P_{j_{i_{r}}^{r}}\right) \cdot\left(h_{1} P_{j_{1}^{0}} \cdots h_{1} P_{j_{i_{0}}^{0}}\right) v^{(n)}, \quad j_{1}^{m} \geq \cdots \geq j_{i_{m}}^{m},
$$

with $i_{0}+\cdots+i_{r}=n$.

The analog of Proposition [10] is straightforwardly similar. Repeating the proof of Proposition [1] let us show that these monomials span the Weyl module. 
By Lemma 2 we have the restriction $i_{1}+\cdots+i_{r} \leq n$. Let us fix the grading, that is fix the integers $i_{1}, \ldots, i_{r}$ (but not $i_{0}$ ). Similarly introduce the partial order comparing $i_{0}$, and then the number of $j_{k}^{r}$ equal to one.

Then each monomial (7) can be obtained as the highest monomial in

$$
\left(e_{1, r+1} P_{j_{1}^{0}} \cdots e_{1, r+1} P_{j_{i_{0}}^{0}}\right) \cdot f_{1, r+1}^{i_{0}} \cdot\left(f_{12} P_{j_{1}^{1}} \cdots f_{12} P_{j_{i_{1}}^{1}}\right) \cdots\left(f_{1, r+1} P_{j_{1}^{r}} \cdots f_{1, r+1} P_{j_{i_{r}}^{r}}\right) v^{(n)}
$$

with respect to this order. So for $i_{0}+i_{1}+\cdots+i_{n}>n$ each monomial (7) is the highest monomial of a relation, therefore by Lemma 5 (ii) the other monomials (7) span the module. For $i_{0}+i_{1}+\cdots+i_{n}<n$ we just set the additional indexes $j_{s}^{0}$ equal to one.

Define the action of $S^{n}(A)$ and the ideal $J_{\{\mathcal{Z}\}_{n \omega_{1}}}$ as above.

Theorem 7. For any $\{\mathcal{Z}\}_{n \omega_{1}}$ we have the natural isomorphism of $s l_{r+1} \otimes A$-modules

$$
W_{M}\left(\{\mathcal{Z}\}_{n \omega_{1}}\right) \cong S^{n}\left(L_{A}\left(V_{r+1}\right)\right) / J_{\{\mathcal{Z}\}_{n \omega_{1}}} \cdot S^{n}\left(L_{A}\left(V_{r+1}\right)\right)
$$

Proof. Straightforwardly similar to Theorem 4

In the case of an arbitrary Lie algebra $\mathfrak{g}$ the following analog of the conjecture $\mathrm{CP}$ about global Weyl modules looks reasonable.

Conjecture 1. Let $V^{(i)}, i=1 \ldots r$ be the fundamental representations of $\mathfrak{g} . \quad B y v^{(i)} \in V^{(i)}$ denote the highest weight vectors. Then the module $W_{A}(\lambda)$ over the algebra $\mathfrak{g} \otimes A$ is isomorphic to the submodule of

$$
\left(V^{(1)} \otimes A\right)^{\otimes \lambda_{1}} \bigotimes \cdots \bigotimes\left(V^{(r)} \otimes A\right)^{\otimes \lambda_{r}}
$$

generated by $\left(v^{(1)} \otimes 1\right)^{\otimes \lambda_{1}} \otimes \cdots \otimes\left(v^{(r)} \otimes 1\right)^{\otimes \lambda_{r}}$.

We also expect some results similar to $\mathrm{CK}]$.

\section{Case of polynomial algebra}

3.1. General case. Recall that by Corollary 1 any local Weyl module on a non-singular variety of dimension $d$ is isomorphic to the tensor product of some modules $W_{\mathbb{C}^{d}}\left(\{0\}_{\lambda^{i}}\right)$. Let us describe them for $\mathfrak{g}=s l_{r+1}$ and the weight equal to $n \omega_{1}$ in more detail.

Let $M=\mathbb{C}^{d}$, then $A=\mathbb{C}\left[x^{1}, \ldots, x^{d}\right]$. We will write it as $A=\mathbb{C}[\mathbf{x}]$, where $\mathbf{x}=\left(x^{1}, \ldots, x^{d}\right) \in \mathbb{C}^{d}$. Also fix the notation $\mathbb{C}\left[\mathbf{x}_{1}, \ldots, \mathbf{x}_{n}\right]$ for the algebra of polynomials in $n$ multivariables (that is in $d n$ usual variables) and the notation $\mathbb{C}\left[\mathbf{x}_{1}, \ldots, \mathbf{x}_{n}\right]_{+}$for the ideal of polynomials with zero at the origin.

Note that the symmetric group $\Sigma_{n}$ acts on $\mathbb{C}\left[\mathbf{x}_{1}, \ldots, \mathbf{x}_{n}\right]$ by permutation of multivariables. Then the space of invariants $\mathbb{C}\left[\mathbf{x}_{1}, \ldots, \mathbf{x}_{n}\right]^{\Sigma_{n}}$ generalizes the notion of symmetric polynomials.

Introduce the Frobenius transformation $\mathcal{F}_{n}^{r+1}$ from the Grothendieck ring of the symmetric group $\Sigma_{n}$ to the Grothendieck ring of $s l_{r+1}$ mapping any $\Sigma_{n}$-module $\pi$ to the $s l_{r+1}$-module $\left(V_{r+1}^{\otimes n} \otimes \pi\right)^{\Sigma_{n}}$. Here $s l_{r+1}$ acts on the factors $V_{r+1}$, and $\Sigma_{n}$ acts on $V_{r+1}^{\otimes n}$ by permuting the factors as well as on $\pi$.

To be explicit, $\mathcal{F}_{n}^{r+1}$ maps the irreducible representation of $\Sigma_{n}$ corresponding to a partition $\xi$ to the irreducible representation of $s l_{r+1}$ with highest weight $\left(\xi_{1}-\xi_{2}, \ldots, \xi_{r}-\xi_{r+1}\right)$ if $\xi_{r+2}=0$ and to zero otherwise.

For an algebra $A$ and a subalgebra $B$ introduce the notation $A / \cdot B=A /(B \cdot A)$.

Corollary 2. For $\mathfrak{g}=s l_{r+1}$ we have an isomorphism of $s l_{r+1}$-modules

$$
W_{\mathbb{C}^{d}}\left(\{0\}_{n \omega_{1}}\right) \cong \mathcal{F}_{n}^{r+1}\left(\mathbb{C}\left[\mathbf{x}_{1}, \ldots, \mathbf{x}_{n}\right] / \cdot \mathbb{C}\left[\mathbf{x}_{1}, \ldots, \mathbf{x}_{n}\right]_{+}^{\Sigma_{n}}\right) .
$$

Proof. Note that $A^{\otimes n} \cong \mathbb{C}\left[\mathbf{x}_{1}, \ldots, \mathbf{x}_{n}\right]$ and $S^{n}(A) \cong \mathbb{C}\left[\mathbf{x}_{1}, \ldots, \mathbf{x}_{n}\right]^{\Sigma_{n}}$. Therefore

$$
S^{n}\left(L_{A}\left(V_{r+1}\right)\right) \cong\left(V_{r+1}^{\otimes n} \otimes \mathbb{C}\left[\mathbf{x}_{1}, \ldots, \mathbf{x}_{n}\right]\right)^{\Sigma_{n}}
$$

and $J_{\{0\}_{\mathrm{n} \omega_{1}}} \cong \mathbb{C}\left[\mathbf{x}_{1}, \ldots, \mathbf{x}_{n}\right]_{+}^{\Sigma_{n}}$. So by Theorem $\mathbf{Z}$ the quotient $W_{\mathbb{C}^{d}}\left(\{0\}_{n \omega_{1}}\right)$ is exactly as proposed.

Also let us calculate weights multiplicities of this module.

Corollary 3. For $\mathfrak{g}=s l_{r+1}$ we have

$$
W_{\mathbb{C}^{d}}\left(\{0\}_{n \omega_{1}}\right)^{\left(i_{0}-i_{1}, i_{1}-i_{2}, \ldots, i_{r-1}-i_{r}\right)} \cong \mathbb{C}\left[\mathbf{x}_{1}, \ldots, \mathbf{x}_{n}\right]^{\Sigma_{i_{0}} \times \cdots \times \Sigma_{i_{r}}} / \cdot \mathbb{C}\left[\mathbf{x}_{1}, \ldots, \mathbf{x}_{n}\right]_{+}^{\Sigma_{n}},
$$

where $i_{0}+\cdots+i_{r}=n$ and $\Sigma_{i_{0}} \times \cdots \times \Sigma_{i_{r}}$ acts as a subgroup of $\Sigma_{n}$.

Proof. It follows in the similar way from Theorem 7 and Proposition 13

So investigation of Weyl modules in this case is reduced to a question about the multi-variable analog of symmetric functions. 
3.2. One-dimensional case. This calculation is based on the following statement.

Theorem 8. (Chevalley) The representation of $\Sigma_{n}$ in the quotient $\mathbb{C}\left[x_{1}, \ldots, x_{n}\right] / \cdot \mathbb{C}\left[x_{1}, \ldots, x_{n}\right]_{+}^{\Sigma_{n}}$ is isomorphic to the regular one.

In $[\mathrm{CP}]$ it is shown that in $s l_{2}$ case we have

$$
\operatorname{dim} W_{\mathbb{C}}\left(\{0\}_{n \omega}\right)=2^{n}, \quad \operatorname{dim} W_{\mathbb{C}}\left(\{0\}_{n \omega}\right)^{n-2 i}=\left[\begin{array}{l}
n \\
i
\end{array}\right] .
$$

Let us slightly generalize this statement.

Proposition 14. For $\mathfrak{g}=s l_{r+1}$ we have

$$
\operatorname{dim} W_{\mathbb{C}}\left(\{0\}_{n \omega_{1}}\right)=(r+1)^{n}, \quad \operatorname{dim} W_{\mathbb{C}}\left(\{0\}_{n \omega_{1}}\right)^{\left(i_{0}-i_{1}, i_{1}-i_{2}, \ldots, i_{r-1}-i_{r}\right)}=\frac{n !}{i_{0} ! i_{1} ! \ldots i_{r} !}
$$

for all $i_{0}+\cdots+i_{r}=n$.

Proof. Let us prove the second formula, then the first one follows. By Corollary 3 we have

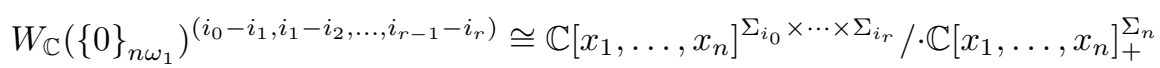

So we have the subspace of invariants in the regular representation $\mathbb{C}\left[\Sigma_{n}\right]$ with respect to the subgroup $\Sigma_{i_{0}} \times$ $\cdots \times \Sigma_{i_{r}}$. Then the dimension is equal to the index of the subgroup.

3.3. Two-dimensional case. This calculation is based on the following result from $\mathrm{H}$.

Recall that a function $f:\{1, \ldots, n\} \rightarrow\{1, \ldots, n\}$ is called a parking function if $\left|f^{-1}(\{1, \ldots, k\})\right| \geq k$ for $k=1 \ldots n$.

The group $\Sigma_{n}$ acts on the set $P F_{n}$ of parking functions by permutation on the domain. Let $\mathbb{C} P F_{n}$ be the permutation representation of $\Sigma_{n}$ on parking functions.

Theorem 9. (See $[\mathrm{H}]$, Theorem 3.10 together with Proposition 3.13) Let $\mathbf{x}_{i} \in \mathbb{C}^{2}$. Then the representation of $\Sigma_{n}$ in the quotient $\mathbb{C}\left[\mathbf{x}_{1}, \ldots, \mathbf{x}_{n}\right] / \cdot \mathbb{C}\left[\mathbf{x}_{1}, \ldots, \mathbf{x}_{n}\right]_{+}^{\Sigma_{n}}$ is isomorphic to the product $\mathbb{C P} F_{n} \otimes \varepsilon$, where $\varepsilon$ is the sign representation of $\Sigma_{n}$.

To describe $\mathbb{C} P F_{n}$ more explicitly introduce the class of integer sequences

$$
\mathcal{A}_{n}=\left\{\left(a_{1}, \ldots, a_{n}\right) \mid a_{i} \geq 0, a_{1}+\cdots+a_{n}=n, a_{1}+\cdots+a_{k} \geq k \text { for } k=1 \ldots n\right\} .
$$

Proposition 15. We have

$$
\mathbb{C} P F_{n}=\bigoplus_{\mathcal{A}_{n}} \operatorname{Ind} \sum_{\Sigma_{a_{1}} \times \cdots \times \Sigma_{a_{n}}}^{\Sigma_{n}} \mathbb{C}
$$

Proof. For a sequence $\left(a_{1}, \ldots a_{n}\right)$ with $a_{i} \geq 0, a_{1}+\cdots+a_{n}=n$ consider the set of functions $f:\{1, \ldots, n\} \rightarrow$ $\{1, \ldots, n\}$ such that $\left|f^{-1}(\{i\})\right|=a_{i}, i=1 \ldots n$. This set admits the action of $\Sigma_{n}$ by permutation on the domain and forms the permutation representation isomorphic to

$$
\mathbb{C}\left[\Sigma_{n} / \Sigma_{a_{1}} \times \cdots \times \Sigma_{a_{n}}\right] \cong \operatorname{Ind} \sum_{\Sigma_{a_{1}} \times \cdots \times \Sigma_{a_{n}}}^{\Sigma_{n}} \mathbb{C}
$$

To complete the proof note that parking functions just correspond to $\left(a_{1}, \ldots a_{n}\right) \in \mathcal{A}_{n}$.

By $\mathcal{R}_{n}^{s}$ denote the set of subsets $H \subset\{1 \ldots s n\}$ such that

$$
|H|=n, \quad|H \cap\{1, \ldots, k\}| \geq \frac{k}{s} .
$$

Such subsets are usually called Raney sequences (see GKP], [S]).

Theorem 10. Let $\mathfrak{g}=s l_{r+1}$.

(i) The dimension of the space $W_{\mathbb{C}^{2}}\left(\{0\}_{n \omega_{1}}\right)$ is equal to the number of elements in $\mathcal{R}_{n+1}^{r+1}$.

(ii) The dimension of the space $W_{\mathbb{C}^{2}}\left(\{0\}_{n \omega_{1}}\right)^{\left(i_{0}-i_{1}, i_{1}-i_{2}, \ldots, i_{r-1}-i_{r}\right)}$ is equal to the number of elements $H \in \mathcal{R}_{n+1}^{r+1}$ such that

$$
|\{i \in H \mid i \equiv j+1 \bmod (r+1)\}|=i_{j}, j=1 \ldots r
$$


Proof. By Corollary 2 and Theorem 9 we have

$$
W_{\mathbb{C}^{2}}\left(\{0\}_{n \omega_{1}}\right) \cong \mathcal{F}_{n}^{r+1}\left(\mathbb{C}\left[\mathbf{x}_{1}, \ldots, \mathbf{x}_{n}\right] / \cdot \mathbb{C}\left[\mathbf{x}_{1}, \ldots, \mathbf{x}_{n}\right]_{+}^{\Sigma_{n}}\right) \cong \mathcal{F}_{n}^{r+1}\left(\mathbb{C} P F_{n} \otimes \varepsilon\right),
$$

so the Frobenius duality and Proposition 15 imply

$$
\begin{aligned}
& W_{\mathbb{C}^{2}}\left(\{0\}_{n \omega_{1}}\right) \cong\left(V_{r+1}^{\otimes n} \otimes \mathbb{C} P F_{n} \otimes \varepsilon\right)^{\Sigma_{n}} \cong \operatorname{Hom}_{\Sigma_{n}}\left(\varepsilon \otimes\left(V_{r+1}^{*}\right)^{\otimes n}, \mathbb{C} P F_{n}\right) \cong \\
& \cong \bigoplus_{\mathcal{A}_{n}} \operatorname{Hom}_{\Sigma_{n}}\left(\varepsilon \otimes\left(V_{r+1}^{*}\right)^{\otimes n}, \operatorname{Ind}_{\Sigma_{a_{1}} \times \cdots \times \Sigma_{a_{n}}} \mathbb{C}\right) \cong \bigoplus_{\mathcal{A}_{n}} \operatorname{Hom}_{\Sigma_{a_{1}} \times \cdots \times \Sigma_{a_{n}}}\left(\varepsilon \otimes\left(V_{r+1}^{*}\right)^{\otimes n}, \mathbb{C}\right) \cong \bigoplus_{\mathcal{A}_{n}} \bigotimes_{i=1}^{n}\left(\wedge^{a_{i}} V_{r+1}\right) .
\end{aligned}
$$

Let $v$ be the highest weight vector of $V_{r+1}$. Choose the graded basis $v_{j} \in V_{r+1}, j=1 \ldots r+1$, by setting $v_{j}=f_{1, j+1} v$ for $j \leq r$ and $v_{r+1}=v$. Then the graded monomials spanning $W_{\mathbb{C}^{2}}\left(\{0\}_{n \omega_{1}}\right)$ look like

$$
\left(v_{j_{1}^{1}} \wedge \cdots \wedge v_{j_{a_{1}}^{1}}\right) \bigotimes \cdots \bigotimes\left(v_{j_{1}^{n}} \wedge \cdots \wedge v_{j_{a_{n}}^{n}}\right), \quad 1 \leq j_{t}^{s} \leq r+1, \quad\left(a_{1}, \ldots, a_{n}\right) \in \mathcal{A}_{n} .
$$

With this element we associate the set

$$
H=\left\{1,(r+1)(s-1)+j_{t}^{s}+1 \mid t=1 \ldots a_{s}, s=1 \ldots n\right\} .
$$

Then the condition $H \in \mathcal{R}_{n+1}^{r+1}$ is equivalent to $\left(a_{1}, \ldots, a_{n}\right) \in \mathcal{A}_{n}$. So we have a bijection between $\mathcal{R}_{n+1}^{r+1}$ and graded monomials. Concerning the grading note that the number of factors $v_{j}$ for $j=1 \ldots r$ in a graded monomial is equal to the number of elements in the corresponding set $H$ equivalent to $j+1$ modulo $r+1$.

Corollary 4. For $\mathfrak{g}=s l_{2}$ we have

$$
\operatorname{dim} W_{\mathbb{C}^{2}}\left(\{0\}_{n \omega}\right)=\frac{(2 n+2) !}{(n+1) !(n+2) !}
$$

that is the Catalan numbers and

$$
\operatorname{dim} W_{\mathbb{C}^{2}}\left(\{0\}_{n \omega}\right)^{n-2 i}=\frac{(n+1) ! n !}{(n-i+1) !(n-i) !(i+1) ! i !}, \quad i=0 \ldots n,
$$

that is the Narayana numbers.

Proof. Let $C_{0}(z)=z^{-1}$ and

$$
C_{n+1}(z)=\sum_{i=0}^{n} z^{n-2 i} \cdot \frac{(n+1) ! n !}{(n-i+1) !(n-i) !(i+1) ! i !} .
$$

Then we have (see $\underline{\underline{S}}$, Section 6)

$$
C_{n+1}(z)=\sum_{s=0}^{n} C_{s}\left(z^{-1}\right) \cdot C_{n-s}(z) .
$$

To prove the corollary it is enough to check this relation for

$$
\operatorname{ch} W_{\mathbb{C}^{2}}\left(\{0\}_{n \omega}\right)=\sum_{i=0}^{n} z^{n-2 i} \cdot \operatorname{dim} W_{\mathbb{C}^{2}}\left(\{0\}_{n \omega}\right)^{n-2 i} .
$$

By Theorem 10 we can work with Raney sequences, namely let $R_{n}^{(i)}$ be the number of sets $H \in \mathcal{R}_{n}^{2}$ such that $H$ has exactly $i$ even elements. Let $R_{0}(z)=z^{-1}$ and

$$
R_{n+1}(z)=\sum_{i=0}^{n} z^{n-2 i} \cdot R_{n+1}^{(i)} .
$$

Then it is enough to show that $R_{n+1}(z)=\sum_{s=0}^{n} R_{s}\left(z^{-1}\right) \cdot R_{n-s}(z)$.

Take $H \in \mathcal{R}_{n+1}^{2}$. Let $s$ be the first non-negative integer such that $|H \cap\{1, \ldots, 2 s+2\}|=s+1$. Then we can split $H$ into

$$
H_{1}=\{i \in 1 \ldots 2 s \mid i+1 \in H\}, \quad H_{2}=\{i \in 1 \ldots 2 n-2 s \mid i+2 s+2 \in H\} .
$$

Clearly $H_{1} \in \mathcal{R}_{s}^{2}$ and $H_{2} \in \mathcal{R}_{n-s}^{2}$. On the other hand, if $H_{1} \in \mathcal{R}_{s}^{2}$ and $H_{2} \in \mathcal{R}_{n-s}^{2}$ then we can glue them into the set

$$
H=\{1\} \cup\left\{i+1 \mid i \in H_{1}\right\} \cup\left\{i+2 s+2 \mid i \in H_{2}\right\} \in \mathcal{R}_{n+1}^{2}
$$

Concerning the grading note that the set of even elements in $H$ is formed by odd elements in $H_{1}$ and even elements in $H_{2}$. And the inversion of the formal variable $z$ in the recurrence relation means exactly that we count odd elements instead of even ones. 
Corollary 5. For $\mathfrak{g}=s l_{r+1}$ we have

$$
\operatorname{dim} W_{\mathbb{C}^{2}}\left(\{0\}_{n \omega_{1}}\right)=\frac{((r+1)(n+1)) !}{(n+1) !(r(n+1)+1) !} .
$$

That is the higher Catalan numbers.

Proof. Similar to $s l_{2}$ case. See GKP], Section 7.5 concerning Raney sequences.

So the weight multiplicities of these modules can be considered as higher Narayana numbers.

\subsection{A conjecture for higher dimensions.}

Conjecture 2. For $\mathfrak{g}=s l_{2}$ we have

$$
\operatorname{dim} W_{\mathbb{C}^{d}}\left(\{0\}_{n \omega}\right)^{n-2 i}=\frac{(d+n-1) ! \cdots(d+n-i) !}{(n-1) ! \cdots(n-i) !} \cdot \frac{2 ! \cdots(i-1) !}{d ! \cdots(d+i-1) !}, \quad i=0 \ldots n,
$$

that equal to the dimension of the irreducible representation of $s_{n}$ with highest weight $d \omega_{i}$ for $i=1 \ldots n-1$ (and equal to one for $i=0, n)$.

Then the dimensions of Weyl modules $W_{\mathbb{C}^{d}}\left(\{0\}_{n \omega}\right)$ form so called Hoggatt sequences (see [FA]).

\section{APPENDIX}

Here we perform a formal computation used in this paper. We take a commutative algebra $A$ with unit and consider the Lie algebra $s l_{2} \otimes A$. Let $P_{1}, \ldots P_{n} \in A$, and suppose that the vector $v$ is such that $\left(e \otimes P_{i}\right) v=$ $\left(h \otimes P_{i}\right) v=0$ for all $i$. Let us write $f$ instead of $f \otimes 1$. Our aim is to calculate

$$
\left(e \otimes P_{1}\right) \ldots\left(e \otimes P_{n}\right) f^{m} v .
$$

Suppose we have $m$ indistinguishable glasses ( $\left.\operatorname{set} \mathcal{G}_{m}\right)$ and $n$ distinguishable ingredients $\left(I_{1}, \ldots, I_{n}\right)$. By cocktail serving denote a way $S$ to distribute ingredients between glasses (each ingredient into only one glass). By $\mathcal{S}(m, n)$ denote the set of all possible cocktail serving.

We now associate an element of $U\left(s l_{2} \otimes A\right)$ with a set of elements $P_{1} \ldots, P_{n} \in A$ and a cocktail serving $S$. First for each glass $G \in \mathcal{G}_{m}$ we take $P(G) \in A$ equal to the product of $P_{i}$ for all $I_{i}$ from the glass $G$. Then we set $f(S)=\prod_{G \in \mathcal{G}_{m}}(f \otimes P(G))$.

Proposition 16. We have

$$
\left(e \otimes P_{1}\right) \ldots\left(e \otimes P_{n}\right) f^{n+m} v=\sum_{S \in \mathcal{S}(m, n)} c(S) f(S) v,
$$

where $c(S)$ are integers and $(-1)^{n} c(S)>0$ for $m>0$.

Proof. Induction on $n$. For $n=0$ we have empty glasses and the only constant equal to one. Concerning the step, note that

$$
\begin{array}{r}
(e \otimes P)\left(f \otimes Q_{1}\right) \ldots\left(f \otimes Q_{n}\right) v=\sum_{i=1}^{n}\left(f \otimes Q_{1}\right) \ldots\left(f \otimes Q_{i-1}\right)\left(h \otimes P Q_{i}\right)\left(f \otimes Q_{i+1}\right) \ldots\left(f \otimes Q_{n}\right) v= \\
=-2 \sum_{i<j}\left(f \otimes P Q_{i} Q_{j}\right) \prod_{k \neq i, j}\left(f \otimes Q_{k}\right) v=-\sum_{i \neq j}\left(f \otimes P Q_{i} Q_{j}\right) \prod_{k \neq i, j}\left(f \otimes Q_{k}\right) v .
\end{array}
$$

For $G_{1}, G_{2} \in \mathcal{G}_{m}$ introduce the mixing map $\operatorname{Mix}_{n+1}^{G_{1}, G_{2}}: \mathcal{S}(m, n) \rightarrow \mathcal{S}(m-1, n+1)$ that identifies the glass $G_{1}$ with the glass $G_{2}$, puts there all their ingredients and finally adds there the ingredient $I_{n+1}$. Then for any $S \in \mathcal{S}(m, n-1)$ we have

$$
\left(e \otimes P_{n}\right) f(S) v=-\sum_{G_{1} \neq G_{2} \in \mathcal{G}_{m}} f\left(\operatorname{Mix}_{n}^{G_{1}, G_{2}}(S)\right) v .
$$

By induction we have (8), but it remains to show that $(-1)^{n} c(S)>0$. Note that $c(S)$ is the sum of $-c\left(S^{\prime}\right)$ such that $S^{\prime} \in \mathcal{S}(m+1, n-1)$ and $S$ is obtained from $S^{\prime}$ by mixing. Therefore $(-1)^{n} c(S) \geq 0$ and we have to show that the mixing map is surjective. Consider $S^{\prime}$ obtained from $S$ as follows. We remove the glass $G$ containing $I_{n}$ and instead take the glasses $G_{1}$ and $G_{2}$ containing the other (excluding $I_{n}$ ) ingredients from $G$. Then we have $S=\operatorname{Mix}_{n}^{G_{1}, G_{2}}\left(S^{\prime}\right)$. 


\section{REFERENCES}

[CK] Vyjayanthi Chari, Michael Kleber, Symmetric Functions and Representations of Quantum Affine Algebras, Infinite dimensional Lie theory and conformal field theory, Charlottesville, May 2000; math.QA/0011161

[CL] V. Chari, T. Le, Representations of Double Affine Lie algebras; math.QA/0205312

[CP] V. Chari, A. Pressley, Weyl Modules for Classical and Quantum Affine Algebras, Represent. Theory 5 (2001), 191-223; math.QA/0004174

[FA] D. C. Fielder and C. O. Alford, An investigation of sequences derived from Hoggatt sums and Hoggatt triangles, in G. E. Bergum et al., editors, Applications of Fibonacci Numbers: Proc. Third Internat. Conf. on Fibonacci Numbers and Their Applications, Pisa, Jul 25-29, 1988. Kluwer, Dordrecht, Vol. 3, 1990, pp. 77-88.

[FF] B. L. Feigin and E. B. Feigin, q-characters of the tensor products in sl 2 -case, Moscow Math. Journal 2 (2002), no. 3, 567-588; math.QA/0201111

[FL] B. Feigin, S. Loktev, On Generalized Kostka Polynomials and the Quantum Verlinde Rule, Differential topology, infinite-dimensional Lie algebras, and applications, Amer. Math. Soc. Transl. Ser. 2, Vol. 194 (1999), p. 61-79; math.QA/9812093

[GKP] R. Graham, D. Knuth, O. Patashnik, Concrete Mathematics, Addison-Wesley, 1998.

[H] M. Haiman, Vanishing theorems and character formulas for the Hilbert scheme of points in the plane, Invent. Math. 149, no. 2 (2002) 371-407; math.AG/0201148

[K] V. Kac, Infinite dimensional Lie algebras, Cambridge Univ. Press, 1985.

[S] R. Stanley, Enumerative Combinatorics, Cambridge Studies in Advanced Mathematics, V.62.

BF: Landau institute for Theoretical Physics, Chernogolovka 142432, Russia

E-mail address: feigin@feigin.mccme.ru

SL: Institute for Theoretical and Experimental Physics, B. Cheremushkinskaja, 25, Moscow 117259, Russia

Independent University of Moscow, B. Vlasievsky Per., 11, Moscow 121002, Russia

E-mail address: loktev@mccme.ru 